\title{
URÓBORO': LA SERPIENTE QUE SE MUERDE LA COLA EN LOS TEXTOS ALQUÍMICOS GRIEGOS
}

\author{
Aurelio J. Fernández García \\ IES Viera y Clavijo \\ afergar@gmail.com
}

\begin{abstract}
RESUMEN
Uno de los símbolos más característicos de las mitologías de las civilizaciones antiguas es Uróboro, la serpiente que se muerde la cola. Su figura y los textos alquímicos que hablan de ella se encuentran en dos de los principales manuscritos que se describen en el Catalogue des Manuscrites Alquimiques: el Marcianus graecus 299 y el Parisinus graecus 2327. En este artículo se ofrece el texto griego con su traducción, además de un breve comentario, de los pasajes de estos manuscritos en los que aparece este símbolo alquímico.
\end{abstract}

PALABRAS CLAVE: alquimia, serpiente, símbolo, naturaleza, manuscrito.

\section{ABSTRACT}

"Ouroboros, the snake that bites its tail in alchemical Greek texts». One of the most characteristic symbols of the mythologies of the old civilizations is Ouroboros, the snake that bites its tail. Its figure and the alchemical texts that speak of it are in two of the main manuscripts that are described in the Catalogue des Manuscrites Alquimiques: the Marcianus graecus 299 and the Parisinus graecus 2327. This article offers the Greek text with its translation, in addition to a brief commentary, of the passages of these manuscripts in which this alchemical symbol appears.

KEY WORDS: alchemy, snake, symbol, nature, manuscript.

El lenguaje simbólico² fue una característica común de las mitologías de Oriente Medio y de la India ${ }^{3}$; de las religiones mistéricas del mundo antiguo, incluyendo Grecia y Roma; de la magia popular; e incluso del cristianismo primitivo y del gnosticismo. De una forma especial, la simbología tuvo un desarrollo especial en la mayoría de los textos antiguos de la alquimia griega, constituyendo, con toda probabilidad, una de sus señas de identidad más relevante.

Sin lugar a dudas, uno de esos símbolos más representativos es la serpiente que se muerde la cola. Las representaciones más tempranas de este motivo ${ }^{4}$, en relación con la antigua alquimia griega, se encuentran en el Papiro V y en el Papiro W de Leiden ${ }^{5}$; sin embargo, parecen estar más en relación con la magia ${ }^{6}$ que con 
aquella, puesto que recuerdan las sencillas inscripciones en gemas gnósticas en las que el nombre de «Abraxas`» está rodeado por la serpiente que se muerde la cola ${ }^{8}$ y que solían ser utilizadas como talismán.

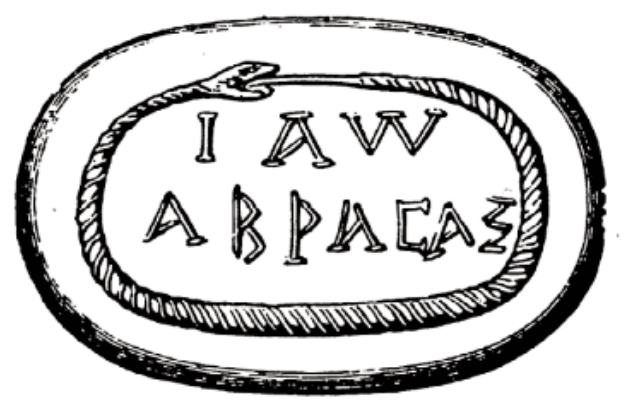

(Figura 1. Ejemplo de gema gnóstica. Sheppard, 1962: 85)

Uno de los primeros testimonios escritos en el dominio alquímico, en el que se cita la serpiente que se muerde la cola, es el de Olimpiodoro (s. V o s. VI) ${ }^{9}$ que

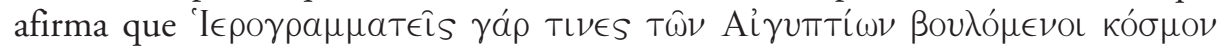

1 Transcripción de oủpoßópos, -ov: adjetivo de dos terminaciones que significa «que se muerde la cola».

${ }^{2}$ Sobre la fenomenología de los símbolos, véase Aladro Vico, 2010.

${ }^{3}$ La serpiente que se muerde la cola está atestiguada en Mesopotamia: véase Kakosy, 1986 : col. 889; y Déonna, 1952: 168. Sin embargo, es en Egipto, sobre todo, donde se testimonia su empleo desde época muy antigua. Se menciona por primera vez en los Textos de las Pirámides: Hornung, 1992: 145, 161-162 y 272; y sus primeras representaciones se remontan a la XVIII dinastía, constatándose claramente sobre uno de los capiteles dorados de Tutankamón: véase Piankoff, 1951: pl. IV.

${ }^{4}$ Véase Neumann, 1954: 49, que dice que este motivo se encuentra en todas las edades y culturas; y Forster y Simon, 2008: 172, que dicen que este símbolo se encuentra en todo el mundo.

${ }^{5}$ Véase Berthelot, 1889: 9, 18.

${ }^{6}$ Este motivo se atestigua también en los PGM (abreviación de Papyri Graecae Magicae): véase Mertens, 1995: 179. Para más información sobre el empleo de esta divinidad en los PGM, véase Diblasi Neto, 2015.

${ }^{7}$ Abraxas, que en letras, por el valor numérico de estas, corresponde al número mágico de los días del año: $1+2+100+1+60+1+200=365$. Este nombre se relaciona, por otro lado, con la palabra "Abracadabra», carente de sentido.

${ }^{8}$ Véase Berthelot ,1885: 62. La serpiente que se muerde la cola fue un símbolo característico de algunas sectas gnósticas como los ofitas y dentro de estos, los naasenos: Bianchi, 1978: 254; y Rudolph, 1983: 89. Los gnósticos, así como los primeros alquimistas y los neoplatónicos de Alejandría, unían la magia a sus prácticas religiosas.

${ }^{9}$ Berthelot y Ruelle, 1888: II, 80, 9-11; y Berthelot, 1885: 192 ss. 


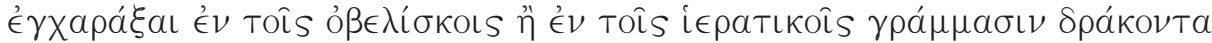

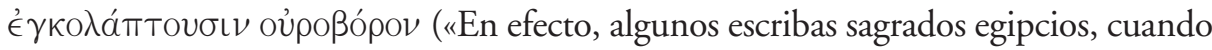
quieren representar el universo en los obeliscos o en los escritos sagrados, graban una serpiente que se muerde la cola $»^{10}$ ).

La figura de la serpiente que se muerde la cola y los textos alquímicos que hablan de ella se encuentran en dos de los principales manuscritos que se describen en el Catalogue des Manuscrites Alquimiques. el Marcianus graecus 299 (=M, s. X o s. XI) y el Parisinus graecus 2327 (=A, s. XV) $)^{11}$.

\section{EL MARCIANUS GRAECUS 299 (=M)}

Este manuscrito, copiado sobre pergamino y considerado por la crítica especializada el más antiguo y hermoso de los manuscritos alquímicos ${ }^{12}$, contiene 196 folios de entre veintinueve y treinta líneas en cada uno de ellos y distribuidos en veinticuatro cuadernos.

Para el tema que nos ocupa, nos interesa el folio $188 \mathrm{v}$ que forma parte de una serie de pasajes atribuidos a Zósimo de Panópolis ${ }^{13}$, con la denominación de

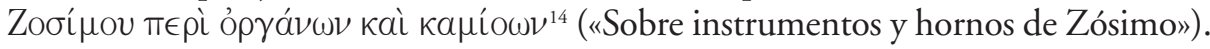

En este folio se puede ver un grupo de cinco figuras:

${ }^{10}$ Los egipcios representaron la eternidad, es decir, el tiempo infinito, como una serpiente devorando su propia cola. El poeta Claudiano, nacido en Alejandría, escribió en los últimos cincuenta versos de su poema Consulado de Estilión II: vv. 424 y sigs. un pasaje bastante intrigante, al indicar que el Sol, tras uncir un carro, visita la caverna del Tiempo (Aíw): «Existe lejos, desconocida e impenetrable para nuestra raza, apenas accesible para los dioses, una caverna de inmensa edad, tenebrosa madre de los años, que de su anchuroso seno suelta el tiempo y lo hace volver de nuevo. Rodea la cueva una serpiente que todo lo va consumiendo con plácida majestad, perpetuamente mantiene el brillo en sus escamas y con su boca devora la cola curvada hacia atrás volviendo a pasar con el silencioso movimiento por su propio comienzo» (trad. Castillo Bejarano, 1993: 107-108).

${ }^{11}$ Véase Mertens, 1995: XXII-XXIX y XXXI-XXXVIII, respectivamente.

${ }_{12}$ Véase Mertens, 1995: XXII: n. 45.

${ }^{13}$ Para una revisión de las obras de Zósimo que aparecen en los cuatro manuscritos principales de alquimia griega Marcianus graecus 299 (=M), Parisinus graecus 2325 (=B), Parisinus graecus 2327 (=A) y Laurentianus graecus 86, 16 (=L): véase Mertens, 1995: XLIII-LXIX. Cf. igualmente Mertens, 1995: CVI ss. la crítica que hace a la edición de los escritos de este autor griego de Berthelot y Ruelle, 1967: 107-250.

${ }^{14}$ Folios 186r-188v. 


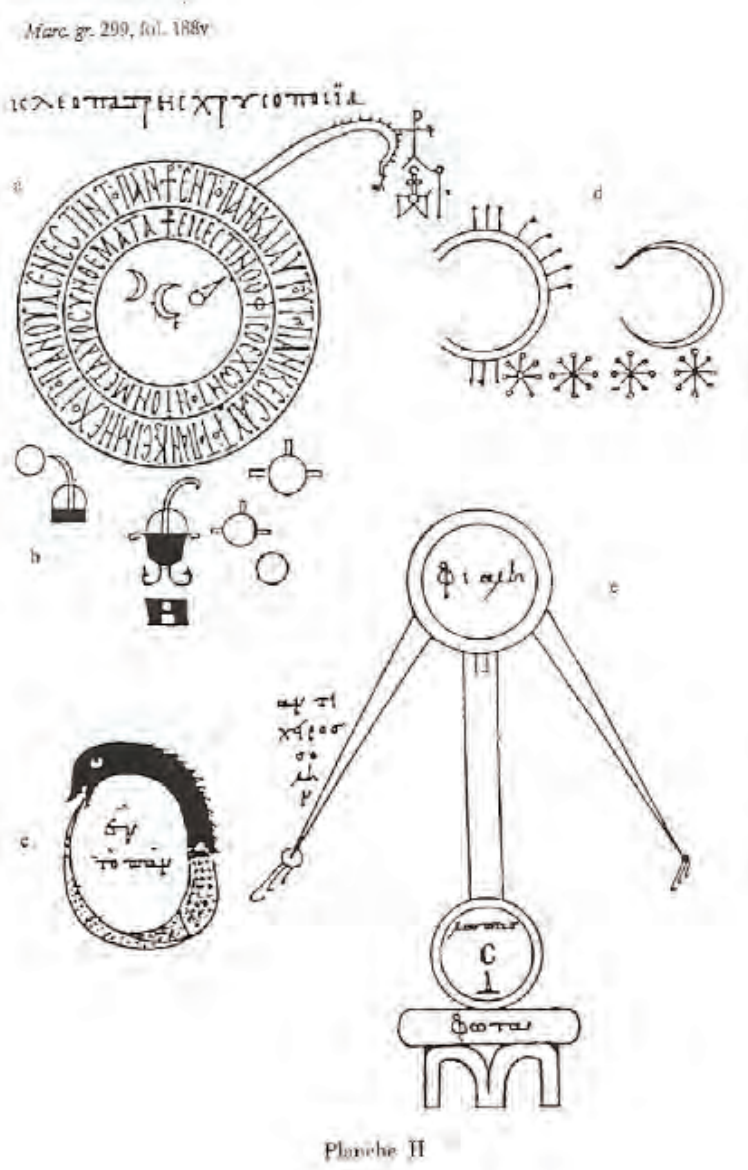

(Figura 2. «Crisopea de Cleopatra». Mertens, 1995: 241)

El dibujo a) de la figura 2, que se encuentra en la parte superior izquierda

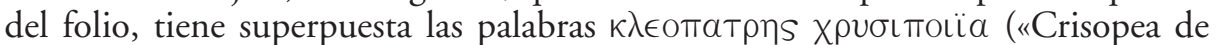
Cleopatra»), por lo que ha llevado a los historiadores de la alquimia a designar a este grupo de figuras (diagramas y aparatos alquímicos) con la denominación general de Crisopea de Cleopatra ${ }^{15}$.

${ }^{15}$ Con la denominación de Cleopatra circulaban en la Antigüedad escritos médicos, mágicos y alquímicos. Las figuras en cuestión no parece que tengan ninguna relación con el contenidos de los textos relativos a Cleopatra, en cambio, sí corresponden a los escritos de Zósimo de Panópolis. Para Mertens, 1995: 177, estas palabras no tienen ninguna justificación para estar ahí y deben ser consideradas como un error del copista. De los cuatro manuscritos principales de alquimia griega donde se encuentran las obras de este escritor citados anteriormente, solo es en $\mathrm{M}$ donde se citan estas palabras. 
Este dibujo consta de tres círculos o anillos concéntricos que encierran los axiomas místicos. En el del centro están los símbolos del oro, la plata y el mercurio.

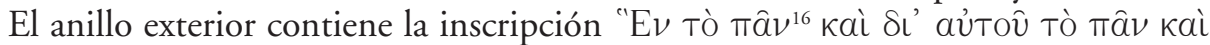

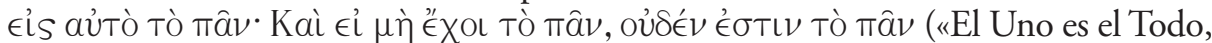
por él es el Todo, y hacia él se dirige el Todo; y si no contuviera el Todo, no existiría

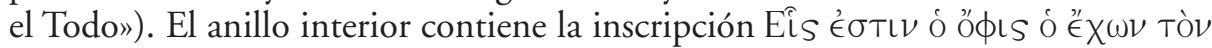
iòv $\mu \in T \grave{\alpha}<T \grave{\alpha}>\delta$ ó $\sigma v \nu \theta \in ́ \mu \alpha T \alpha$ ( $\mathrm{El}$ Uno es la serpiente que contiene la herrumbre después de dos operaciones $\left.{ }^{17} »\right)$. Finalmente, en el lado derecho se extiende una prolongación en forma de cola, acabada en una serie de signos mágicos, que se desarrollan por todas partes. Cabría entender esta figura como una forma complicada y expresiva de serpiente que se muerde la cola.

En el centro de la figura se distinguen tres símbolos: a la izquierda el de la luna creciente orientada al occidente, que representa el mercurio; en el centro, el de la luna creciente orientada al oriente, con una $\epsilon$ en la parte inferior, que representa la plata; y a la derecha el del sol, que representa el oro. Son las tres sustancias que intervienen en la Memoria auténtica n. ${ }^{\circ} \mathrm{V}$ de Zósimo de Panópolis ${ }^{18}$. Las inscripciones interiores de los anillos constituyen el texto de la Memoria auténtica $\mathrm{n} .^{\circ}$ VI de este autor.

Es especialmente interesante para el presente trabajo el dibujo c) de este grupo. Representa a la perfección una serpiente que se muerde la cola. Su cuerpo está dividido cromáticamente en dos partes: una negra y otra blanca que está recubierta de escamas y subdividida, a su vez, en tres zonas asimétricas. En el centro de la figura está la inscripción êv Tò $\pi \hat{a} \nu^{19}$ («el Uno es el Todo»).

El dibujo b) representa un serie de instrumentos alquímicos, el d) son seis dibujos (parece claro que el de la derecha representa el signo del mercurio) y el e) es un «díbico», en el que se distinguen una serie de términos, propios del instrumental

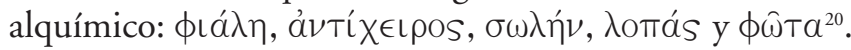

${ }^{16}$ Las palabras "Ev Tò $\pi a ̂ v$, «El Uno y el Todo», aparecen como título del libro 5. de los Ptolemaicos (PGM, XIII, 980).

${ }^{17}$ Se debe entender «operaciones alquímicas».

${ }^{18}$ Véase Mertens, 1995: 22. Para este investigador, Zósimo es el primer alquimista en hacer uso de la serpiente que muerde la cola: 180 . Dentro de los ya citados cuatro manuscritos principales de alquimia griega, las Memorias auténticas de Zósimo se sitúan en M, en los folios 189r-196v; en B, en los folios 82r-88r; en A, en los folios 80r-88v; y en L, en los folios 83v-95v.

${ }^{19}$ Sobre la fórmula êv Tò Tâv , véasen Mertens, 1995: 181 ss. Para la posible influencia estoica de esta leyenda, véase Sheppard, 1962: 192.

${ }^{20} \Phi \iota \alpha ́ \lambda \eta$ es una especie de platillo que tenía un agujero por el que pasan los elementos más volátiles de las sustancias que se destilan; $\sigma \omega \lambda \eta \eta ́ v$ es un tubo ascendente por donde pasan los elementos más volátiles (vendría a ser lo que se llama en la actualidad «cuello de cisne»); $\alpha \nu \tau i ́ \chi \in L \rho O S$ es un tubo inverso al anterior, por donde se recoge el destilado; 入oтás es un matraz de barro o caldera donde se coloca la mezcla de lo que se va a destilar; y $\phi \hat{\omega} \tau a$ es cualquier fuente de calor. $C f$. Fernández García, 2014: 1008 ss. 


\section{EL PARISINUS GRAECUS 2327 (=A)}

Este manuscrito, escrito en papel que lleva las armas del rey Enrique II y proveniente de la biblioteca de Fontainebleau, tiene 299 folios de veintiséis líneas de media, en cada uno de ellos ${ }^{21}$. Es un tipo de enciclopedia alquimista, donde el copista ha unido todos los tratados y fragmentos congéneres de los que se tiene conocimiento.

En este manuscrito la figura de la serpiente que se muerde la cola aparece en dos ocasiones, dibujadas y coloreadas con el mayor de los cuidados: folios 196r y 279r. A continuación de cada figura hay un texto.

En el folio 196r el dibujo de la serpiente está compuesta por tres círculos o anillos concéntricos: de colores rojo, amarillo y verde, similares a los de la figura a) de la Crisopea de Cleopatra que se citó anteriormente. Se presenta llena de escamas con tres orejas y cuatro patas. La cabeza, las orejas y el anillo exterior están pintados en rojo vivo (color azafrán); el ojo es de color blanco con la pupila negra. El segundo anillo (el del medio) es de color amarillo. Finalmente, el anillo interior es de color verde, igual que las patas.

Según se describe en el texto del manuscrito, las cuatro patas representan los cuatro elementos: agua, tierra, aire y fuego; es decir, constituyen la tetrasomía ${ }^{22}$. Las tres orejas hacen referencia a los «tres vapores sublimados»: azufre, mercurio y arsénico.

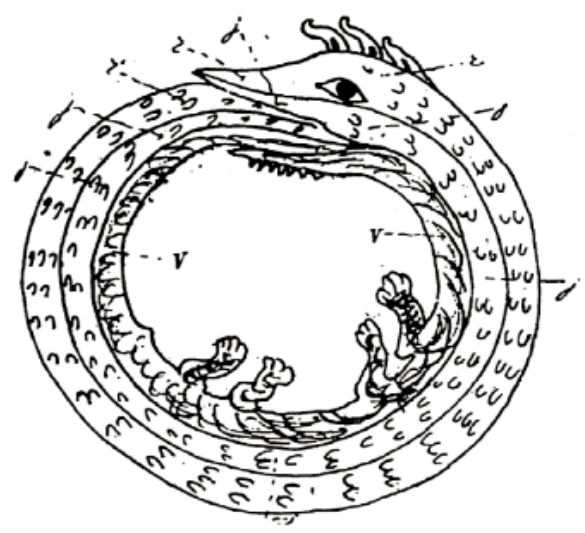

(Figura 3. Uróboro. Sheppard, 1962: 85)

${ }^{21}$ En su colofón se indica que fue copiado por Teodoro Pelecano de Corfú. Véase Mertens 1995: XXXI ss.

${ }^{22}$ Los alquimistas retoman la vieja teoría griega de los cuatro elementos, en especial de Aristóteles, no designando de por sí las realidades concretas cuyos nombres llevan, sino estados, modalidades 


\section{Se presentan a continuación el texto griego y su traducción ${ }^{23}$ :}

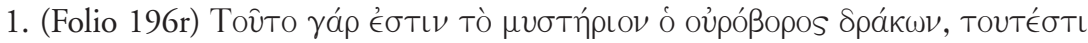

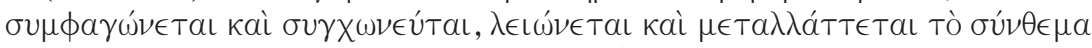

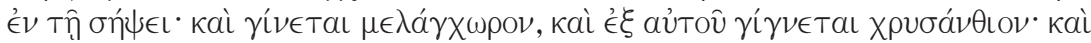

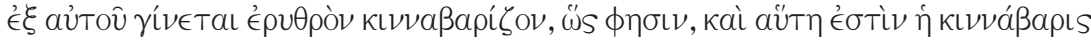

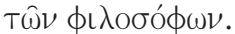

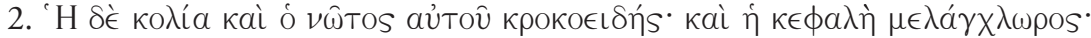

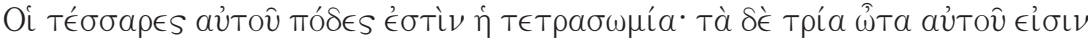

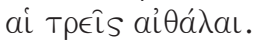

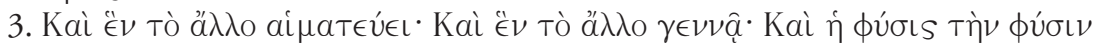

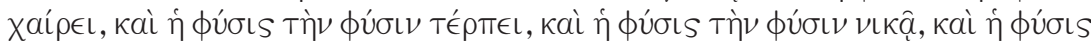

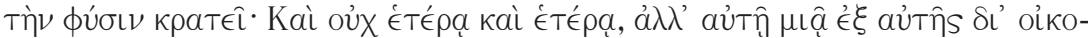

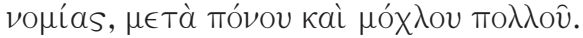

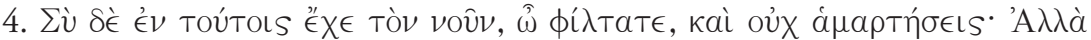

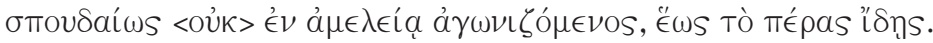

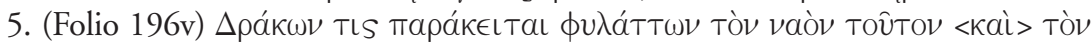

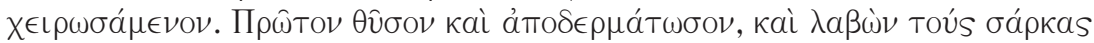

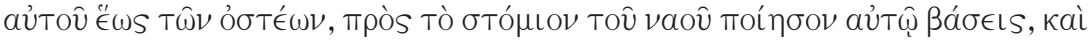

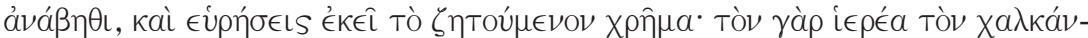

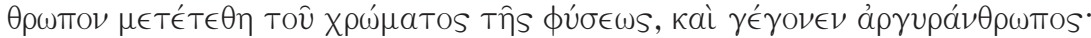

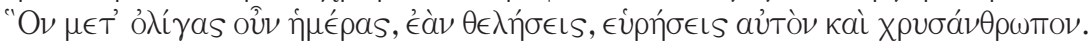
«1. Este es, por tanto, el misterio, la serpiente que se muerde la cola; es decir, la composición es devorada, fundida, triturada y modificada por la fermentación; adquiere un color verde oliva oscuro, de este color se pasa a uno dorado y de este, a un color rojo cinabrio, como suele decirse. Este es el cinabrio de los filósofos.

2. Su vientre y espalda son de color azafrán; su cabeza es de color verde oliva oscuro; sus cuatro patas son la tetrasomía; y sus tres orejas son los tres vapores sublimados ${ }^{24}$.

de la materia: el agua es sinónimo del estado líquido; la tierra, del estado sólido; el aire, del estado gaseoso; y el fuego, del estado sutil. Cf. Berthelot y Ruelle, 1967: 96, 6-14, donde Olimpiodoro indica

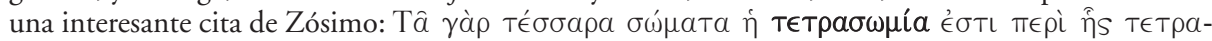

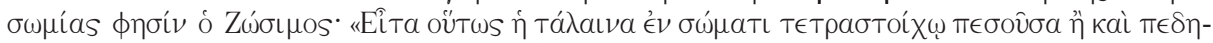

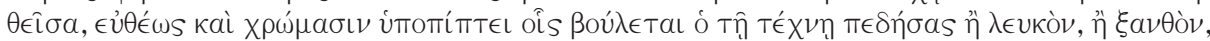

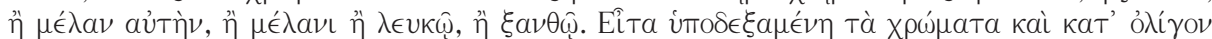

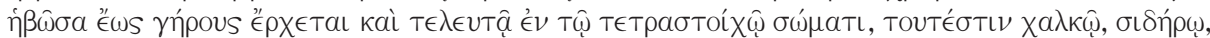

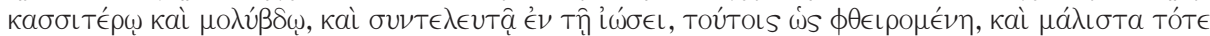

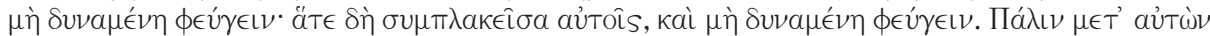

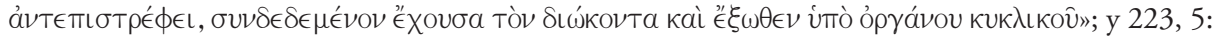

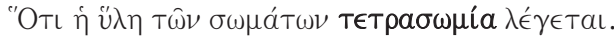

${ }^{23}$ Berthelot y Ruelle, 1967: 21-22.

${ }^{24}$ Se refiere al mercurio, el arsénico y el azufre, sustancias que protagonizan la mayor parte de los textos alquímicos griegos y que son volátiles a temperaturas moderadas. Debido a ello, esas sustancias eran consideradas como "vapores" o "espíritus". 
3. El Uno ${ }^{25}$ da su sangre a lo Otro; el Uno engendra a lo Otro. La naturaleza se complace de la naturaleza, la naturaleza goza de la naturaleza, la naturaleza vence a la naturaleza y la naturaleza domina a la naturaleza. Y no es por causa de una o de otra naturaleza, sino por su propia naturaleza única que procede de sí misma, consecuencia de la operación ${ }^{26}$, con trabajo y mucho esfuerzo.

4. Pon tu inteligencia, muy apreciado amigo, en estos asuntos y no te equivocarás. Pero esfuérzate con seriedad y diligencia, hasta que veas el final ${ }^{27}$.

5. Una serpiente está apostada y vigila el templo que la tiene, además, subyugada. En primer lugar, sacrifícala y despelléjala; vete quitándole su parte carnosa hasta que llegues a sus huesos; pon peanas a la entrada del templo y colócate encima; y allí encontrarás la cosa que buscas. Pues el animal sacrificado, el hombre de cobre, fue cambiando de color por su naturaleza; pasó a convertirse en hombre de plata; y pocos días después, si tú quieres, también en hombre de oro».

En el folio 279r del mismo manuscrito, se encuentra la segunda figura. La acompaña un texto en el que el principio alquímico es diferente, pero con la misma receta mística. La serpiente que se muerde la cola está compuesta, en este caso, por dos círculos o anillos concéntricos: de colores rojo y verde. Aquí las escamas están mejor marcadas. Como la figura que se comentó anteriormente, está provista también de cuatro pies y tres orejas.

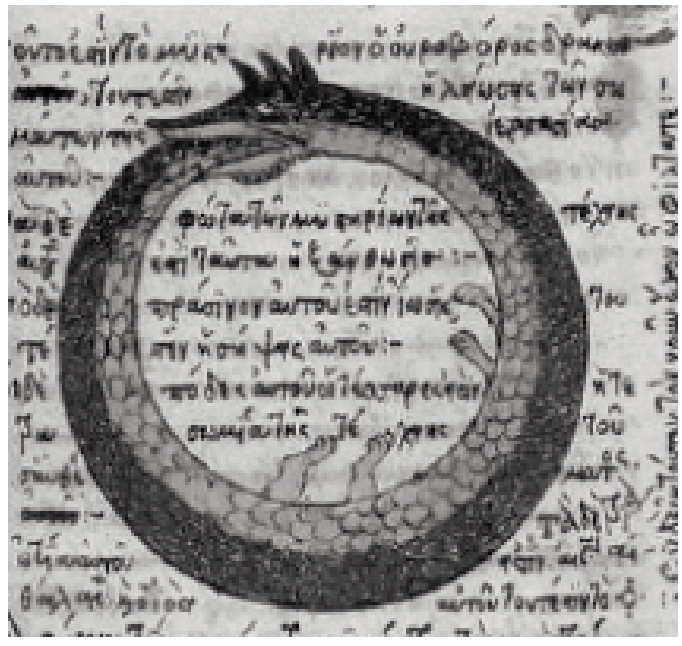

(Figura 4: https://www.flickr.com/photos/ouroboran/2288405597/in/photostream/lightbox ${ }^{28}$ )

${ }^{25}$ La Unidad. La unidad de la materia se representa en numerosas ocasiones con el motivo de la serpiente que se muerde la cola. Es el símbolo de la evolución que renace sin cesar de su propia destrucción, en un movimiento sin fin.

${ }^{26}$ Se refiere a la "operación alquímica».

${ }^{27}$ Se refiere al final del proceso alquímico.

${ }^{28}$ Fecha de la consulta: 21 de abril de 2017. 
Se presentan a continuación el texto griego y su traducción ${ }^{29}$ :

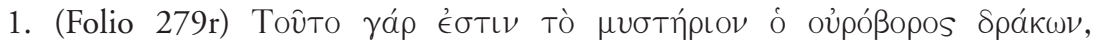

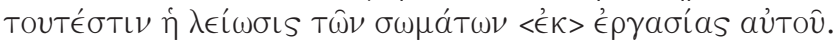

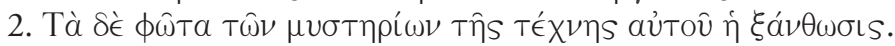

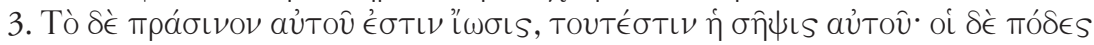

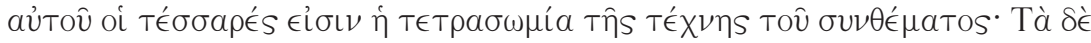

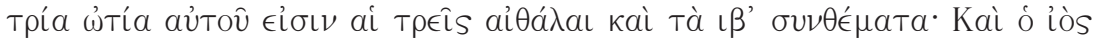

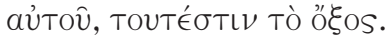

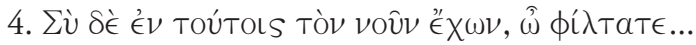

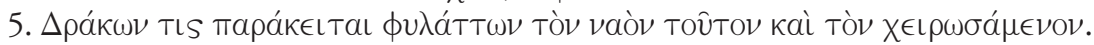

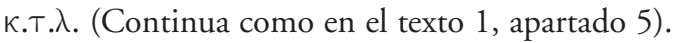

«1. Este es, por tanto, el misterio, la serpiente que se muerde la cola: es decir, la conversión de los cuerpos en polvo, a partir de su operación ${ }^{30}$.

2. Las luces de los misterios del arte ${ }^{31}$ es la coloración en amarillo.

3. Lo verde de la serpiente es oxidación: es decir, su fermentación. Sus cuatro patas son la tetrasomía empleada en la composición del $\operatorname{arte}^{32}$; y sus tres orejas son los tres vapores sublimados y las doce composiciones. Su agente corrosivo, no puede ser nada más que el vinagre.

4. Pon tu inteligencia, muy apreciado amigo...

5. Una serpiente está apostada y vigila el templo que la tiene, además, subyugada, etc.».

Como se dijo anteriormente, la serpiente que se muerde la cola es uno de los símbolos más representativos de la obra alquímica, de la Ars Magna, en el que se entremezclan leyes naturales y conceptos filosóficos.

En efecto, Uróboro, la serpiente que se muerde la cola, sugiere la idea del proceso cíclico de la naturaleza: los ciclos del día y la noche, los de las estaciones de la naturaleza, el movimiento circular de los astros en el cosmos, los periodos de la vida humana: la vida y la muerte..., incluso los ciclos de la historia. Por otro lado, el círculo, la línea curva, siempre fascinó a las civilizaciones antiguas, puesto que como trayectoria continua que se mueve sobre sí misma, no tiene principio ni fin, y representa lo infinito y lo eterno, la infinitud y la totalidad, al mismo tiempo. Expresa la unidad de las cosas, las materiales y las espirituales, que nunca desaparecen ${ }^{33}$.

Incluso Jung, uno de los máximos expertos del simbolismo psicológico, se sirvió del proceso alquímico, en general, y de la serpiente que se muerde la cola, en

${ }^{29}$ Véase Berthelot y Ruelle, 1967: 22-23.

${ }^{30}$ Se refiere a la "operación alquímica».

${ }^{31}$ Se refiere siempre al «arte alquímico».

${ }^{32}$ Véase nota anterior.

${ }^{33}$ Para Koepgen, 1938: 149, el orden circular constituye una característica principal de la forma de pensar gnóstica. 
particular, para establecer la analogía de la problemática de los opuestos, característica de la alquimia, con la disociación de la personalidad en el ámbito psíquico, y poder explicar, de esa manera, los procesos neuróticos y psíquicos del ser humano.

Para Jung, «el Uroboros que se come su propia cola es un símbolo drástico de la asimilación e integración del opuesto, de la sombra. Al mismo tiempo, este proceso circular es explicado como un símbolo de la inmortalidad, es decir, de la constante auto-renovación, pues se dice del uroboros que se mata a sí mismo, se da vida a sí mismo, se fecunda y se da a luz. El uróboros representa desde antiguo lo uno que surge de la unión de lo que está en disputa consigo mismo, por lo que constituye el misterio de la prima materia, que en cuanto proyección procede inequívocamente de lo inconsciente humano $»^{34}$.

\section{REFERENCIAS BIBLIOGRÁFICAS}

Aladro Vico, E. (2010): «La estructura de los símbolos», Perspectivas de la comunicación 3, 2: 134-147. Berthelot, M. (1885): Les Origines de l'Alchimie, París (reimp. 1938).

— (1889): Introduction à l'Étude de la Chimie des Anciens et du Moyen-Âge, París (reimp. 1938).

Berthelot, M. y Ruelle, C. E. (1888): Collection des Anciens Alchimistes Grecs (CAAG), t. II, París (reimp. Osnabrück, 1967).

BIANCHI, U. (1978): Selected Essays on Gnosticism, Leiden.

Castillo Bejarano, M. (1993): Claudiano. Poemas II, Ed. Gredos, Madrid.

DÉOnNa, W. (1952): «Ouroboros», Artibus Asiae 15: 63-170.

Diblasi NeTO, I. (2015): “Quando meus lábios sacerdotais disserem palavras secretas”: Abraxas, magia e política nos papiros mágicos gregos», Romanitas-Revista de Estudos Grecolatinos 5: 131-146.

FERnÁNdeZ GarCía, A. J. (2014): «Instrumentos alquímicos de María la Judía», en MarTínez FernÁNdez, A., Ortega Villarco, B., Velasco López, H., Zamora Salamanca, H. (eds.), Ágalma. Ofrenda desde la Filología clásica a Manuel García Teijeiro, Valladolid, Universidad de Valladolid, pp. 1001-1010.

Forster, M. y Simon, C. (2008): An A to Z of the Occult, Londres.

Hornung, E. (1992): Les Dieux d'Egypte - L'un et le multiple, París.

KAKOSY, L. (1986): «Uroboros», en Lexikon der Ägyptologie, VI, col. 886-893.

Koepgen, G. (1939): Die Gnosis des Christentums, Salzburgo.

JunG, C. G. (1955/1956): Mysterium Coniuctionis. Untersuchungen über die Trennung und Zusammensetzung der seelischen Gegensätze in der Alchemie (20163: Obra completa de Carl Gustav Jung. Volumen 14: Mysterium coniunctionis: investigación sobre la separación y la unión de los

${ }^{34}$ Jung 20163: 353. 
opuestos animicos en la alquimia (1955/1956). Traducción de J. RIVERA DE ROSALES Y J. NAVARRO PÉREZ).

Mertens, M. (1995): Les Alchimistes Grecs, IV. Zosime de Panopolis. Mémoires autentiques, París.

Neumann, E. (1954): The Origins and History of Consciousness, Londres.

Piankoff, A. (1951): Le chapelles de Tout-Ankh-Amon, II, El Cairo.

Preisendanz, K. (1928-1931): Papyri Graecae Magicae, 2 vol., Leipzig-Berlín (reimp. Stuttgart, 1973-1974).

Rudolph, K. (1983): Gnosis: The Nature and History of Gnosticism, Londres.

SHeppard, H. J. (1962): «The Ouroboros and the Unity of Matter in Alchemy: a Study in Origins», Ambix 10: 83-96. 
